\title{
Terminal Olefin Synthesis via Reaction of Aldehydes with Allylsamarium Bromide
}

\section{Key words}

terminal olefines aromatic aldehydes

samarium
Significance: A simple and efficient one-pot protocol for the synthesis of terminal alkenes has been reported. The reaction of carbonyl compounds with allylsamarium bromide in the presence of diethyl phosphate furnishes the corresponding olefins in good to excellent yields.
Comment: Allylsamarium bromide acts in the reported reaction as both, a nucleophilic reagent and a single electron transfer reagent. Moreover, the described methodology is very versatile since several functional groups are tolerated. 